\title{
Distribución espacial y temporal de aves playeras (Orden: Charadriiformes) en Laguna San Ignacio, Baja California Sur, México
}

\author{
Luis Francisco Mendoza \& Roberto Carmona* \\ Departamento Académico de Biología Marina, Universidad Autónoma de Baja California Sur, Apartado postal 19-B, \\ La Paz, Baja California Sur, CP 23080, México; beauty@uabcs.mx, luis_kiwi@hotmail.com \\ * Correspondence: beauty@uabcs.mx
}

\section{Recibido 10-I-2012. Corregido 05-VIII-2012. Aceptado 06-IX-2012.}

\begin{abstract}
Temporal and spatial distribution of shorebirds (Charadriiformes) at San Ignacio Lagoon, Baja California Sur, Mexico. Baja California Peninsula has several wetlands that represent important ecosystems for shorebirds. San Ignacio Lagoon is one of these sites, and supports $10 \%$ of the total abundance of shorebirds reported in this Peninsula. Since there is few information about this group in this area, we studied spatial and temporal changes in abundance and distribution of shorebirds in San Ignacio Lagoon. For this, we conducted twelve monthly censuses (October 2007-September 2008) on the entire internal perimeter of the lagoon, which we divided into four areas: two at the North and two at the South. We observed a seasonal pattern, with the lowest abundance in May (1 585 birds) and the highest in October (47 410). The most abundant species were Marbled Godwits (Limosa fedoa; $55 \%$ of the total records), Western Sandpipers (Calidris mauri; 23\%), and Willet (Tringa semipalmata; 10\%). All three species were more abundant in autumn; for both, the Marbled Godwit and Willet, we observed their highest numbers in winter and spring, while the Western Sandpiper showed noticeable oscillations, reaching a maximum in early winter (December). In summer, Marbled Godwit and Willet were the only birds present but in lower numbers. Here present the first records of the Pacific Red Knot (Calidris canutus roselaari) in the area. Bird abundance and species richness were influenced seasonally by migration and spatially by sites in the lagoon. The greatest shorebird abundance was in the South area of the lagoon, probably because of better accessibility to food. Our results allowed the inclusion of San Ignacio Lagoon in the Western Hemisphere Shorebirds Reserve Network (WHSRN) as a site of international importance. Rev. Biol. Trop. 61 (1): 229-241. Epub 2013 March 01.
\end{abstract}

Key words: shorebirds, migration, Limosa fedoa, San Ignacio Lagoon, Baja California Peninsula, México.

En la región Neártica se localizan las áreas de reproducción de 49 especies de aves playeras (Orden Charadriiformes, Myers et al. 1987). La mayor parte de estas especies son migratorias (Howes \& Bakewell 1989) y al menos 40 de ellas utilizan áreas tropicales y subtropicales de México, Centro y Sudamérica para pasar el invierno (Donaldson et al. 2000, Brown et al. 2001, SEMARTAT 2008).

En el continente Americano, para realizar los movimientos entre sus áreas de reproducción y sus sitios de invernada, las aves playeras utilizan tres principales corredores migratorios: Atlántico, Transcontinental y Pacífico. Este último es el más importante por el número de especies y de individuos que lo utilizan (Myers et al. 1987). A lo largo de este corredor existen diferentes humedales utilizados como zonas de paso y como áreas de invernada (Page et al. 1997, Kelly 2001, Stenzel et al. 2002, Carmona et al. 2004).

A través del tiempo ha crecido el interés internacional en las áreas de paso y sitios de invernada (Tsipoura \& Burger 1999, Skagen et al. 2003), donde las aves playeras se concentran en grandes números y presentan las mayores tasas de mortalidad (Shepherd et al. 2003). Este interés surge debido a la declinación en 
las poblaciones de diferentes especies, atribuida principalmente a la pérdida o degradación de humedales (Erwin et al. 1986, Morrison et al. 2006).

A lo largo de la Península de Baja California, ubicada en el corredor migratorio del Pacífico, existen diferentes humedales utilizados por las aves playeras (Massey \& Palacios 1994); en éstos invernan al menos 355000 individuos (Page \& Palacios 1993, Page et al. 1997). En su porción centro-occidental, dentro de los límites de la Reserva de la Biosfera El Vizcaíno, se ubica Laguna San Ignacio, la cual ha sido reconocida como una de las más importantes de la región para las aves playeras (Page et al. 1997), con cerca del 10\% del total de individuos invernantes en la Península, sólo superada numéricamente por el complejo lagunar Guerrero Negro-Ojo de Liebre y por el Delta del Río Colorado (Page \& Palacios 1993, Page et al. 1997, SEMARNAT 2008).

Pese a la importancia de la Laguna San Ignacio para las aves playeras, existe escasa información sobre estas poblaciones. Los pocos trabajos realizados se circunscriben a descripciones cualitativas que proporcionan información limitada sobre la distribución de las aves dentro de la laguna (Huey 1927, Danemann \& Guzmán-Poo 1992). Las publicaciones que incluyen estimaciones de abundancia son puntuales en el tiempo, pues se realizaron sólo con información de invierno (Morrison et al. 1992, Page \& Palacios 1993, Page et al. 1997), lo que no ha permitido conocer el patrón de utilización temporal de la zona por parte de las diferentes especies de aves playeras; información requerida para detectar las preferencias y los posibles cambios en el uso de los diferentes ambientes de la zona, datos que ayudarían a establecer las bases de programas de conservación. Por lo anterior, el trabajo presente es el primero en hacer un seguimiento anual de la abundancia y distribución de aves playeras en Laguna San Ignacio.

\section{MATERIALES Y MÉTODOS}

Laguna San Ignacio está localizada en la costa centro-occidental de la Península de Baja
California, México, dentro de los límites del área protegida más grande del país, la Reserva de la Biosfera El Vizcaíno (Fig. 1; Danemann \& Guzmán-Poo 1992). La laguna presenta profundidades de $2-4 \mathrm{~m}$ en la mayor parte de su extensión, que llegan hasta los $26 \mathrm{~m}$ en los canales que la comunican con el Océano Pacífico. Se compone de dos grandes brazos: uno hacia el sudeste, que presenta grandes extensiones de mangle (Rhizophora mangle y Laguncularia racemosa, Carabias et al. 2000) y canales poco profundos, y otro con mayor superficie que se orienta hacia el norte, donde predominan playas arenosas y salitrales. En la línea de costa de la laguna existen playas arenosas y limosas, manglares, parches de costa rocosa, conchales y marismas (Danemann \& GuzmánPoo 1992). La marea semidiurna mixta expone grandes planicies lodosas (Danemann \& Guzmán-Poo 1992) y alcanza una amplitud cercana a $3 \mathrm{~m}$ verticales.

El perímetro interno del área de estudio se dividió en cuatro zonas (Fig. 1), dos

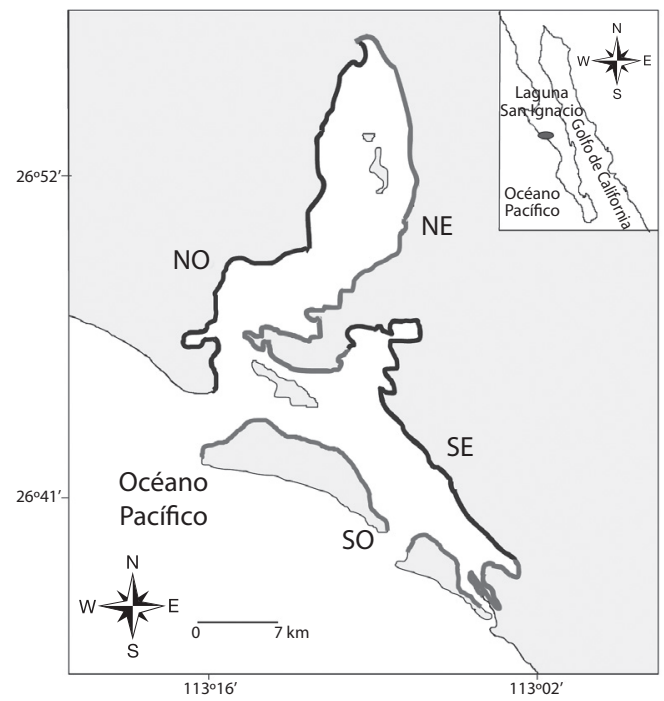

Fig. 1. Área de estudio. Se resaltan las zonas de monitoreo en las que se dividió Laguna San Ignacio. (NE=noreste, $\mathrm{NO}=$ noroeste, $\mathrm{SE}=$ sureste, $\mathrm{SO}=$ suroeste).

Fig. 1. Study area. There are marked monitoring areas in which was divided the San Ignacio Lagoon. (NE=Northeast, $\mathrm{NO}=$ Northwest, $\mathrm{SE}=$ Southeast, $\mathrm{SO}=$ Southwest). 
correspondientes al brazo norte: noroeste (NO) y noreste (NE) y dos al sur: suroeste (SO) y sureste (SE). El criterio fundamental para la división norte y sur fue la presencia de bosques de manglar, que predominan en esta última, mientras que las divisiones este y oeste se basaron en el tipo de sustrato, pues es general en la costa oeste predominaron playas arenosas. La zona SO se caracteriza por presentar áreas extensas de manglar y marismas, y donde la bajamar exhibe planicies lodosas amplias y canales de marea largos, su línea de costa mide alrededor de $38 \mathrm{~km}$. La zona SE presenta áreas con manglar y marismas, con predominio de sustratos lodosos con algunos parches de salitral y áreas arenosas, durante la marea baja se observan planicies lodosas que sirven como áreas de alimentación a las aves playeras, tiene una longitud de $32 \mathrm{~km}$. La zona NO presenta algunos parches de manglar y marismas. La línea de costa tiene sustratos rocosos, barras y dunas, salitrales extensos y acantilados, su longitud es de $35 \mathrm{~km}$. Por último la zona NE presenta áreas pequeñas con manglar al sur, en contraste al norte se extienden salitrales grandes. La zona intermareal esta formada por sustratos rocosos, playas arenosas y conchales, tiene una longitud de $45 \mathrm{~km}$.

Se realizaron visitas mensuales a cada una de las áreas entre octubre de 2007 y septiembre de 2008. Se llevaron a cabo censos desde una embarcación de $7 \mathrm{~m}$ de eslora con motor fuera de borda de $75 \mathrm{hp}$. Se cubrió, en cada recorrido, la totalidad de las cuatro zonas establecidas y se navegó lo más cercano posible a la línea de costa. Los censos se realizaron, en general, a primeras horas de la mañana con mareas medias ascendentes para facilitar la entrada de la embarcación a diferentes bajos.

Las especies se identificaron mediante guías de campo (Sibley 2000, National Geographic 2002). Se emplearon conteos directos en parvadas de hasta 300 individuos, parvadas mayores se estimaron utilizando el método de conteo por bloques (Howes \& Bakewell 1989), el tamaño del bloque varió de 10-500 individuos. El número de aves en cada bandada fue estimado por dos observadores, de tal forma que si las estimaciones diferían el ejercicio se repetía. Se emplearon para las observaciones binoculares (10x42) y telescopio (15-60x). La duración de los censos varió dependiendo de la cantidad de aves encontradas, sin embargo, típicamente iniciaron a las 08:00h y terminaron a las 14:00h. Todos los censos se realizaron en la tercera semana del mes correspondiente, haciéndolos coincidir con las mareas vivas. Dado el tamaño del área de estudio y el esfuerzo, cada visita tuvo una duración mensual promedio de tres días.

Se indica el listado de especies de aves playeras registradas en San Ignacio, al que se añade su presencia: migratorio, observable una parte del año con poblaciones invernantes; migratorio, con fracciones poblacionales que veranearon en el área; residente, especies de reproducción local y residentes que incrementan sus números con la llegada de individuos migratorios (Carmona 1995, Howell \& Webb 1995). Además, se indica el estatus de protección por el Gobierno mexicano: amenazada y en peligro de extinción (SEMARNAT 2010). Los nombres en español de las especies se tomaron de Escalante et al. (1996).

Se muestran los cambios temporales observados en la riqueza específica y la abundancia para toda la laguna, y adicionalmente se presentan las abundancias mensuales para cada una de las cuatro zonas. Con base en los registros acumulados, se seleccionaron las especies requeridas para conjuntar al menos el $90 \%$ del total, las más abundantes. Éste criterio fue apoyado con la ayuda de curvas de porcentajes de abundancia acumulada. Para las especies seleccionadas, se muestran las abundancias mensuales en cada una de las cuatro zonas de estudio, lo que describe su patrón de uso espacio-temporal.

Dada la naturaleza cualitativa de los criterios de clasificación (meses, zonas y especies), los análisis se llevaron a cabo mediante pruebas de independencia $X^{2}$ (Zar 1999; $\alpha=0.05$, en todos los casos). Así, inicialmente se probó si la abundancia general de las aves (por especie) puede o no considerar independiente del mes de observación (distribución temporal). 
Posteriormente se realizaron pruebas de independencia para cada una de las especies seleccionadas que acumularon el $90 \%$ del total de registros, considerando como criterios de clasificación la zona y el mes de observación. Por último se realizó un análisis semejante al primero, en el que probó si la abundancia general de las aves (por especie) se puede o no considerar independiente de la zona de observación (distribución espacial).

El número de aves mínimo que utilizó la laguna se determinó mediante la suma de los máximos observados para cada especie, independientemente de su mes de registro, lo que evita el reconteo (Carmona et al. 2011).

\section{RESULTADOS}

En Laguna San Ignacio se registró, durante un ciclo anual (octubre 2007 a septiembre 2008), un total de 21 especies de aves playeras, un género (Limnodromus spp.) e híbridos de ostrero (Haematopus palliatus Temminck 1820 y H. bachmani Audubon 1838), los cuales pueden identificarse según patrón de coloración del plumaje (Wehtje 2005; Cuadro 1).
Predominaron las especies migratorias (65\%), seguidas de las residentes (17\%). Tres especies migratorias $(13 \%)$ presentaron fracciones poblacionales en verano y una $(5 \%)$ de reproducción local vio incrementados sus números con la llegada de individuos migratorios (Cuadro 1). Cuatro especies están protegidas por el Gobierno Mexicano, dos catalogadas como amenazadas: el chorlo nevado (Charadrius nivosus Linnaeus 1758) y el ostrero negro (Haematopus bachmani) y dos más en peligro de extinción: el ostrero americano (Haematopus palliatus) y el playero rojizo del Pacífico (Calidris canutus roselaari; Cuadro 1). La riqueza específica presentó pocas variaciones a lo largo del año, con los valores mayores en los períodos de otoño e invierno y los menores en verano (Fig. 2).

La suma de los máximos mensuales observados de cada especie indica que Laguna San Ignacio fue utilizada por al menos 56700 aves playeras a lo largo del ciclo anual estudiado (Cuadro 1). La abundancia mensual presentó oscilaciones marcadas. Los números más altos se presentaron en otoño (octubre) con 47 000 individuos y los más bajos se registraron

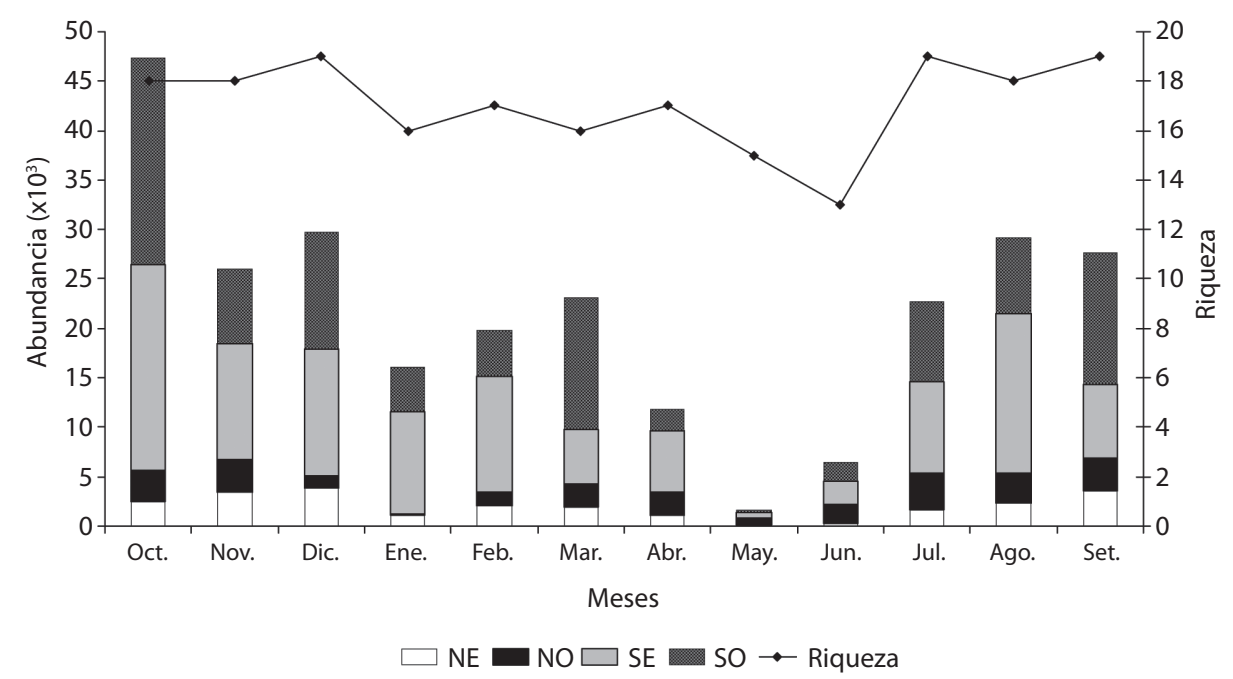

Fig. 2. Riqueza específica (línea) y abundancia (barras) temporales. Las abundancias fueron divididas considerando las cuatro secciones de Laguna San Ignacio ( $\mathrm{NE}=$ noreste, $\mathrm{NO}=$ noroeste, $\mathrm{SE}=$ sureste, $\mathrm{SO}=$ suroeste).

Fig. 2. Specific richness (line) and abundance (bars) per month. Abundances were divided considering the four sections at San Ignacio Lagoon (NE=Northeast, $\mathrm{NO}=$ Northwest, $\mathrm{SE}=$ Southeast, $\mathrm{SO}=$ Southwest). 
CUADRO 1

Aves playeras observadas en Laguna San Ignacio durante la temporada 2007-2008

TABLE 1

Shorebirds observed at San Ignacio Lagoon during the season 2007-2008

\begin{tabular}{|c|c|c|c|c|c|}
\hline Especies & Registros totales & Conteo máximo & Mes de máx. conteo & Presencia & NOM-059 \\
\hline Pluvialis squatarola & 3743 & 865 & Septiembre & M & \\
\hline Charadrius nivosus* & 39 & 17 & Junio & RI & A \\
\hline Charadrius wilsonia* & 4 & 4 & Febrero & $\mathrm{R}$ & \\
\hline Charadrius semipalmatus & 72 & 22 & Diciembre & M & \\
\hline Haematopus palliatus* & 1573 & 287 & Octubre & $\mathrm{R}$ & $\mathrm{P}$ \\
\hline Haematopus bachmani & 92 & 20 & Noviembre & $\mathrm{R}$ & A \\
\hline Haematopus híbrido & 52 & 17 & Julio & $\mathrm{R}$ & \\
\hline Recurvirostra americana & 275 & 72 & Febrero & M & \\
\hline Actitis macularius & 23 & 7 & Septiembre & M & \\
\hline Tringa melanoleuca & 981 & 208 & Septiembre & M & \\
\hline Tringa semipalmata & 27576 & 3486 & Noviembre & MV & \\
\hline Tringa flavipes & 288 & 203 & Enero & M & \\
\hline Numenius phaeopus & 157 & 46 & Julio & M & \\
\hline Numenius americanus & 7109 & 1108 & Marzo & MV & \\
\hline Limosa fedoa & 144167 & 33652 & Octubre & MV & \\
\hline Arenaria interpres & 13 & 9 & Julio & M & \\
\hline Arenaria melanocephala & 1 & 1 & Septiembre & M & \\
\hline Calidris canutus & 1112 & 387 & Febrero & M & $\mathrm{P}$ \\
\hline Calidris alba & 572 & 312 & Abril & M & \\
\hline Calidris mauri & 61755 & 13248 & Diciembre & M & \\
\hline Calidris minutilla & 53 & 33 & Agosto & M & \\
\hline Calidris alpina & 1740 & 405 & Febrero & M & \\
\hline Limnodromus spp. & 10240 & 2352 & Octubre & M & \\
\hline Estimado mínimo & & 56761 & & & \\
\hline
\end{tabular}

* indica especies que se reproducen en el área.

Presencia: $\mathrm{M}=$ migratorio, $\mathrm{MV}=$ Migratorio con fracción veraneante, $\mathrm{R}=$ residente, $\mathrm{R} I=$ residentes que incrementa sus números con individuos migratorios.

NOM-059: estatus de protección según la NOM-059, A=amenazada, P=peligro de extinción.

* indicate species with local reproduction.

Presence: $\mathrm{M}=$ migratory, $\mathrm{MV}=$ migratory with individuals over-summering $\mathrm{R}=$ residents, $\mathrm{RI}=$ resident species increased with migratory individuals.

NO-059: NOM-059 protection status, $\mathrm{A}=$ threatened, $\mathrm{P}=$ endangered.

en verano (mayo) con 1500 aves (Fig. 2). Octubre fue el mes de máxima abundancia, entre noviembre y abril se observaron números medios y estables, entre abril y junio la abundancia decayó nuevamente, para incrementar a partir de julio (Fig. 2). La prueba estadística corroboró la dependencia temporal de las abundancias de las diferentes especies $\left(X_{242}^{2}=47\right.$ $450, \mathrm{p}<0.01)$.
Especies más abundantes: Las especies más abundantes fueron: el picopando canelo (Limosa fedoa Linnaeus 1758, 55\% de los registros totales), el playero occidental (Calidris mauri Cabanis 1857, 24\%) y el playero pihuiuí (Tringa semipalmata Gmelin 1789, 10\%) (Cuadro 1). Estas tres especies acumularon el $90 \%$ de los registros totales durante el ciclo anual. 
Temporalmente el picopando canelo estuvo representado a lo largo de todo el año y fue la especie que marcó el comportamiento del patrón de abundancia general. Esta especie presentó su abundancia mayor en otoño con máximos en octubre; entre noviembre y abril los números fueron relativamente estables y altos, a partir de mayo disminuyó su abundancia (Fig. 3a). Existieron diferencias significativas en la utilización espacio-temporal para esta especie $\left(X_{33}^{2}=31408, \mathrm{p}<0.01\right)$. El playero occidental presentó abundancia alta en otoño con un máximo en diciembre (13000 aves), para disminuir notoriamente el resto del invierno, en marzo se presentó un pequeño incremento y alcanzó sus valores más bajos entre abril y junio (Fig. 3b). La prueba estadística corroboró la dependencia espacio-temporal de las abundancias de esta especie $\left(X_{33}^{2}=22478, \mathrm{p}<0.01\right)$.

A lo largo del año el playero pihuiuí fue la especie que presentó los menores cambios en su abundancia, con números elevados entre julio y noviembre y bajos en mayo (Fig. 3c). Pese a lo anterior la prueba estadística
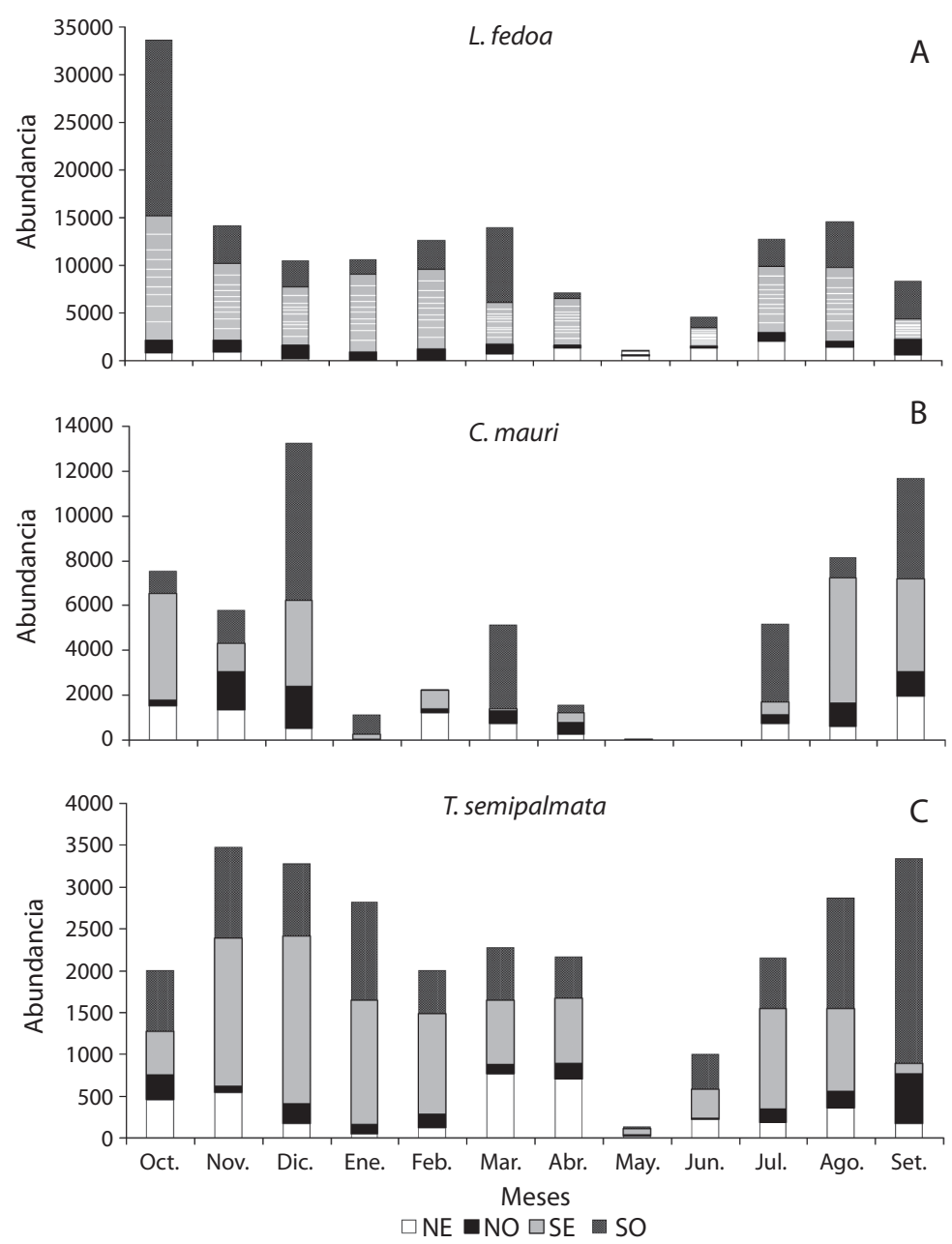

Fig. 3. Cambios temporales en la abundancia de las especies más importantes, considerando las cuatro divisiones de Laguna San Ignacio $(\mathrm{NE}=$ noreste, $\mathrm{NO}=$ noroeste, $\mathrm{SE}=$ sureste, $\mathrm{SO}=$ suroeste).

Fig. 3. Abundances temporal changes of most important species considering the four divisions at San Ignacio Lagoon ( $\mathrm{NE}=$ Northeast, $\mathrm{NO}=$ Northwest, $\mathrm{SE}=$ Southeast, $\mathrm{SO}=$ Southwest). 
evidenció diferencias espacio-temporales de utilización $\left(X_{33}^{2}=6822, \mathrm{p}<0.01\right)$.

Por último, aunque numéricamente no fue una especie abundante, llamó la atención la presencia del playero rojizo del Pacífico (Calidris canutus roselaari Linnaeus 1758). Esta especie tuvo abundancias estables entre octubre y diciembre (de 100-200 aves), en enero se observó una disminución notoria (cuatro individuos), para alcanzar su máximo en febrero (400 aves), entre marzo y agosto presentó sus abundancias menores (de 0-34 aves), por último en septiembre se observó su llegada otoñal (240 aves).

Tendencias espaciales: La abundancia mayor de aves playeras se registró en las dos zonas del brazo sur de la laguna, con $81 \%$ de los registros totales, particularmente en la zona SO (44\%); las zonas del brazo norte presentaron registros menores, con un total de 19\% de los registros (Fig. 2). La prueba estadística corroboró la dependencia espacial de las abundancias de las diferentes especies $\left(X_{66}^{2}=17\right.$ $525, \mathrm{p}<0.01)$. Con respecto a la composición de las especies más abundantes, estas se mantuvieron similares en todas las divisiones.

Se presentaron abundancias altas en el brazo norte de julio a octubre y de marzo a abril; en contraste, las zonas del brazo sur presentaron abundancias más estables. La zona SO mostró picos en otoño (octubre), invierno (diciembre) y primavera (marzo). La zona SE mantuvo sus abundancias altas y constantes en otoño e invierno, para disminuir en primavera y verano (Fig. 2).

El picopando canelo fue la especie predominante en todas las divisiones de la laguna, además fue particularmente abundante en la zona SE (más de 72000 registros); en contraste, la zona NO presentó los registros más pequeños para esta especie (10 000, Fig. 3a). Para el playero occidental los registros mayores se presentaron en la zona SO con 23000 y los menores en la zona NO con 7500 (Fig. 3b). La zona SE presentó los registros mayores del playero pihuiuí con más de 11000 , los registros más bajos (2 000) se presentaron en la zona
NO (Fig. 3c). Se observaron números bajos del playero rojizo del Pacífico en todas las secciones de la laguna, aunque la zona SO fue la más utilizada, en ella se efectuaron 660 registros.

\section{DISCUSIÓN}

Según los últimos estudios y revisiones de diferentes autores, no se observaron dos especies previamente registradas: el candelero americano (Himantopus mexicanus Müller 1776) y el chorlo tildío (Charadrius vociferus Linnaeus 1758; Page \& Palacios 1993, Massey \& Palacios 1994, Page et al. 1997) y se registró una especie que no figuraba en el listado existente para la zona, el playero rojizo del Pacífico (C. canutus roselaari). Las especies ausentes en este estudio han sido registradas como poco comunes y en abundancias bajas para Laguna San Ignacio (Morrison et al. 1992, Page \& Palacios 1993, Page et al. 1997), por lo que se asume que cualitativamente la comunidad de aves playeras está adecuadamente descrita. La riqueza de especies de aves playeras en Laguna San Ignacio es menor a la encontrada en otras lagunas peninsulares, como Ensenada de La Paz que cuenta con 36 especies (Carmona et al. 2008a) y Guerrero Negro con 31 (Carmona et al. 2011). Estas diferencias reflejan en buena medida el esfuerzo de investigación en estos sitios, pues en ambos se han realizado estudios sobre aves playeras por más de cinco años, haciendo que el esfuerzo de observación, en general, incremente las probabilidades de detectar especies poco comunes (Carmona et al. 2008a, Molina et al. 2012).

Los cambios observados en la riqueza y abundancia son producto del movimiento migratorio del $83 \%$ de las especies que utilizaron Laguna San Ignacio (Howell \& Webb 1995). Las riquezas y abundancias mayores observadas en el área de estudio entre otoño e invierno, están relacionadas con el paso migratorio y la invernada, mientras que los valores bajos observados en verano corresponden a la temporada reproductiva, cuando la mayoría de las aves se encuentran en el norte del continente (Myers et al. 1987, O'Reilly \& Wingfield 
1995). Se han registrado comportamientos similares en otros humedales de la Península (Carmona \& Carmona 2000, Danemann et al. 2002, Zárate-Ovando et al. 2006, Carmona et al. 2011).

Previamente, en el sistema lagunar se han reportado abundancias que van de 16402 a 31 500 aves playeras invernantes (Morrison et al. 1992, Page \& Palacios 1993, Page et al. 1997), en contraste, en el trabajo presente se reporta una abundancia de al menos 56700 aves playeras. Estas diferencias pueden estar relacionadas a dos factores: la temporalidad de los censos, pues los trabajos anteriores se limitaron a temporadas de invierno y como se observó en el presente, las abundancias máximas de algunas especies, como el picopando canelo, no necesariamente se alcanzaron en esta época; y a la metodología llevada a cabo, pues pese a las ventajas de los censos aéreos, entre las que se cuenta poder recorrer áreas amplias en poco tiempo, en general esta metodología incrementa los riesgos de subestimación (Briggs et al. 1985, Frederick et al. 2003).

Especies abundantes: La población total de picopando canelo se estima en 170000 individuos (Melcher et al. 2006, Morrison et al. 2006). Según estas estimaciones Laguna San Ignacio es utilizada por $20 \%$ de dicha población como sitio de paso (octubre) y un 9\% de la misma invernó en el área de estudio (noviembre-marzo). Esto convierte a esta laguna en el segundo sitio en importancia en la Península de Baja California, sólo precedido por Guerrero Negro, donde inverna el $50 \%$ del total poblacional (Ayala-Pérez 2010, Carmona et al. 2011).

Respecto a la abundancia temporal del picopando canelo, esta especie se caracterizó por encontrarse bien representada la mayor parte del año, incluso en el verano. La llegada de los primeros individuos en la migración de otoño se observó en julio, lo que coincide con las aves que presentan una partida temprana de los sitios de reproducción (Gratto-Trevor 2000). La llegada numéricamente más importante se observó en octubre, acorde con lo reportado para el arribo a los sitios de invernada (Gratto-Trevor 2000). Sin embargo, a diferencia de Guerrero Negro, que es utilizado fundamentalmente como sitio de invernada (Ayala-Pérez 2010, Carmona et al. 2011), en Laguna San Ignacio una fracción importante utilizó la zona como sitio de paso otoñal. Esta fracción (cerca de 18000 aves) probablemente invernó en humedales peninsulares más sureños, como Bahía Magdalena (ZárateOvando et al. 2006) o la Ensenada de La Paz (Carmona 2007).

La disminución de la abundancia entre marzo y abril se relaciona con la partida de individuos del área de estudio hacia sus sitios de reproducción y coincide con los meses propuestos para la migración primaveral (GrattoTrevor 2000, Ayala-Pérez 2010). Cabe destacar que en primavera no se observaron incrementos importantes en la abundancia, lo que sugiere que las aves que invernaron en sitios sureños de la Península no están utilizando Laguna San Ignacio en su migración al norte. Una fracción de entre 1000-4000 aves veranearon en la zona (entre el 7-27\% de las aves invernantes), esta fracción probablemente esté conformada por aves jóvenes, dado que esta especie se reproduce al segundo o tercer año de edad, pues a estos individuos probablemente les resulte más conveniente permanecer su primer verano en sitios “típicos" de invernada (Gratto-Trevor 2000).

Las abundancias altas del playero occidental en otoño indican que utilizó la zona como área de recuperación en su migración al sur, semejante a lo reportado en otros sitios de la Península (Fernández et al. 1998, Danemann et al. 2002). La abundancia máxima presente en diciembre (13 000 aves) es similar a lo registrado con anterioridad en el área (Page \& Palacios 1993, Page et al. 1997). Sin embargo, llama la atención que a diferencia de otros humedales peninsulares como Guerrero Negro (Carmona et al. 2011) y la Ensenada de La Paz (Galindo 2003), la especie estuvo pobremente representada el resto del invierno, lo que sugiere que Laguna San Ignacio es un sitio por debajo de la calidad promedio para la especie, para corroborarlo son necesarios trabajos de 
marcaje y reobservación, como el realizado en Punta Banda, al norte de la península de Baja California (Fernández et al. 2003).

El playero pihuiuí presentó sus abundancias máximas después de octubre, lo que concuerda con la época en la que las aves abandonan sus áreas norteñas de reproducción (Lowther et al. 2001). La disminución en los números entre abril y mayo concuerda con el inicio de la temporada reproductiva a principios de mayo (Lowther et al. 2001, O'Brien et al. 2006). Las abundancias menores observadas en octubre, al compararlas con las de septiembre y noviembre, pueden relacionarse a movimientos locales, como ha sido sugerido para otras especies (Warnock 1994, Galindo 2003); de hecho, en Guerrero Negro se ha observado un ligero repunte en los números de esta especie en octubre (Carmona et al. 2011). Considerando los registros históricos de la especie en Laguna San Ignacio, al parecer sus abundancias se han mantenido relativamente estables ya que en los últimos 15 años han oscilado entre 2500 (Page \& Palacios 1993, Page et al. 1997) y 3400 (trabajo presente). Esta especie también presentó una fracción veraneante en la zona; de igual forma que lo observado para el picopando canelo, es probable que se trate de individuos jóvenes.

Aunque no fue una de las especies más abundantes, el playero rojizo del Pacífico fue registrado por primera vez para Laguna San Ignacio en el trabajo presente. La subespecie del Pacífico ( $C$. canutus roselaari) está catalogada como en peligro de extinción por el Gobierno mexicano (SEMARNAT 2010). Los censos realizados con anterioridad en la zona no reportan a la especie, probablemente debido al poco esfuerzo temporal; adicionalmente algunos de los censos anteriores se realizaron desde el aire (Page \& Palacios 1993, Page et al. 1997), lo que dificulta la identificación específica, pues buena parte de las aves se catalogan por tamaños (Page \& Palacios 1993). Los números observados entre octubre y diciembre indican la invernada de un pequeño grupo de aves. Es probable que la disminución de enero $\mathrm{y}$ el posterior aumento en febrero se relacionen con movimientos locales, pues en Guerrero Negro invernan entre $6000-7000$ playeros rojizos del Pacífico y es el sitio conocido de mayor importancia para la invernada de esta subespecie (Carmona et al. 2008b, Arce 2011). Las aves vistas en febrero sugieren que Laguna San Ignacio fue utilizada como sitio previo a la migración de primavera, auque abandonaron la zona dos meses antes que las aves de Guerrero Negro (Carmona et al. 2008b). El arribo otoñal también estuvo desfasado (agosto-septiembre), pues en Guerrero Negro se indica un mes antes (Carmona et al. 2008b). En suma, es probable que Laguna San Ignacio sea utilizada como un sitio alterno por las aves de Guerrero Negro, salvo un remanente de 100-200 individuos que invernaron en ella.

Tendencias espaciales: La preferencia por las dos zonas del brazo sur de la laguna puede estar relacionada a la densidad y disponibilidad del alimento (Grant 1984, Yates et al. 1993), lo que a su vez esta determinado por el tipo y características del sedimento (Hsu et al. 2009). Pese a la falta de información, aparentemente las áreas del brazo norte presentan un sedimento más compacto, producto de la menor profundidad y de los largos periodos de desecación, factor que afecta la penetrabilidad del pico de las aves y aumentan el gasto energético para conseguir alimento (Evans 1976, Mouritsen \& Jensen 1992). Adicionalmente se ha observado que en estas zonas hay un predominio de sedimentos arenosos (Álvarez et al. 2002), el cual es un hábitat físicamente riguroso, lo que puede estar ocasionando poca abundancia de invertebrados y presas disponibles (Barnes 1997) y una zona de salitrales amplia, donde aparentemente las salinidades altas impiden el asentamiento de comunidades bentónicas diversas (Verschuren et al. 2000), como las existentes en el brazo sur.

Pese a la presencia baja de aves playeras en las zonas norte, sus abundancias sugieren que estas zonas son importantes durante las épocas migratorias (otoño y primavera), es decir, cuando las aves presentan los mayores 
requerimientos energéticos (O'Reilly \& Wingfield 1995, Tsipoura \& Burger 1999).

Las tres especies más abundantes en Laguna San Ignacio también lo son en otros humedales peninsulares (Page et al. 1997, Zárate- et al. 2006, Carmona et al. 2011). Sin embargo, existen diferencias en las proporciones, ya que en la mayoría de los humedales la especie predominante es el playero occidental (Mellink et al. 1997, Page et al. 1997, Fernández et al. 1998, Danemann et al. 2002, Carmona 2007, Carmona et al. 2011), mientras que para Laguna San Ignacio fue el picopando canelo. Con los datos del trabajo presente y la falta de información general sobre el área de estudio se dificulta explicar estas diferencias. Sin embargo, se ha planteado la posibilidad de que exista una relación entre el tamaño del ave y el tipo de sedimento que frecuenta (Baker 1977). Así las especies grandes como el picopando canelo preferirán humedales con sustratos menos fangosos que las usadas por playeros más pequeños, como el playero occidental. Por comparación los sustratos de Laguna San Ignacio son menos fangosos que los de las otras zonas, lo que permite explicar el predominio de las especies de mayor talla, como el picopando canelo y el playero pihuiuí. Un comportamiento semejante se ha observado localmente en la Ensenada de La Paz, donde zonas con sedimento arenoso son preferidas por el picopando canelo, mientras que en las que predominan sustratos lodosos la especie más abundante es el playero occidental (Carmona 2007). Adicionalmente Bahía Magdalena, sitio donde el picopando canelo es la especie más abundante, se caracteriza por presentar barras, dunas y playas arenosas (Zárate-Ovando et al. 2006, Zárate-Ovando et al. 2008).

En suma, los humedales de Laguna San Ignacio ocupan el tercer lugar en la península por su importancia para las aves playeras; temporalmente la zona es utilizada preferentemente en otoño e invierno y espacialmente es de mayor importancia el brazo sur de la laguna. Los resultados del trabajo presente se enviaron a la Red Hemisférica de Reservas para las Aves Playeras (RHRAP) y permitieron que el área de estudio fuera incluida por dicha organización como Sitio de Importancia Internacional.

\section{AGRADECIMIENTOS}

Este estudio es parte del proyecto titulado "Conservation of the Black Brant and other migratory waterbirds in San Ignacio Lagoon, Mexico" financiado por North American Wetland Conservation Act por medio del Programa México de la División de Conservación de Hábitat para Aves del Servicio de Pesca y Vida Silvestre de los Estados Unidos de América, coordinado por Ellen Murphy. Agradecemos a Pronatura México A.C., en especial a su Director Ejecutivo Gustavo D. Danemann por el apoyo logístico y administrativo durante la realización del estudio presente. Al personal del Laboratorio de Aves acuáticas de la Universidad Autónoma de Baja California Sur por su ayuda en el trabajo de campo, en especial a Israel Martínez y Rosalba Vázquez.

\section{RESUMEN}

Con la pérdida o degradación de humedales han declinado las poblaciones de algunas especies tales como las aves playeras. En vista de que ha crecido el interés internacional por los estudios ecológicos sobre estas especies, se determinó la abundancia, distribución y riqueza espacio-temporal de las aves playeras en Laguna San Ignacio, Península de Baja California. Se realizaron 12 censos mensuales (octubre 2007-septiembre 2008) en el perímetro interno de la laguna; la cual se dividió en cuatro zonas, dos al norte y dos al sur. Temporalmente las abundancias menores se presentaron en mayo (1 585 aves) y las mayores en octubre $(47410)$. Las especies más abundantes fueron: el picopando canelo (Limosa fedoa; 55\% de los registros totales), el playero occidental (Calidris mauri; 23\%) y el playero pihuiuí (Tringa semipalmata; 10\%). Estas especies fueron más abundantes en otoño. El picopando canelo y el playero pihuiuí estabilizaron sus números en invierno y primavera y estuvieron presentes en verano en bajos números, el playero occidental mostró oscilaciones notorias. Se presentan los primeros reportes del playero rojizo del Pacifico (Calidris canutus roselaari) para la zona. La riqueza y abundancia estuvieron influenciadas temporal y espacialmente por las aves migratorias. Las mayores abundancias se presentaron al sur de la laguna, probablemente por la disponibilidad del alimento. Los resultados presentes permitieron incluir al área en la Red 
Hemisférica de Reservas para las Aves Playeras como sitio de importancia internacional.

Palabras clave: aves playeras, migración, Limosa fedoa, Laguna San Ignacio, Península de Baja California.

\section{REFERENCIAS}

Álvarez, S., S. Flores \& V. Sevilla. 2002. Sedimentología y medios ambientes de depósito de Laguna San Ignacio, Baja California Sur, México. Res. XIII Congreso Nacional de Oceanografía. Puerto Vallarta, Jalisco, México.

Arce, N. 2011. Migración e invernada del Playero Rojizo del Pacífico (Calidris canutus roselaari) en Guerrero Negro, Baja California Sur, México. Tesis de Maestría, CICIMAR-IPN, La Paz, BCS., México.

Ayala-Pérez, V. 2010. Patrones de uso durante la época no reproductiva de Limosa fedoa (Aves: Scolopacidae), en Guerrero Negro, Baja California Sur, México. Tesis de Maestría, CICIMAR-IPN, La Paz, BCS., México.

Baker, M.C. 1977. Shorebird food habits in the eastern Canadian Arctic. Condor 79: 56-62.

Barnes, R.S.K (ed.). 1997. The coastline. John Wiley \& Sons, Nueva York, EE.UU.

Briggs, K.T., W.B. Tyler \& D.B. Lewis. 1985. Aerial survey for seabirds: Methodological experiments. J. Wildl. Manage. 49: 412-417.

Brown, S., C. Hicky, B. Harrington \& R. Gill. 2001. United States Shorebird Conservation Plan. Manoment Center for Conservation Sciences.

Carabias, L.J., E. Provencio, E.J. de la Maza, C.D. Gutiérrez, C.M. Gómez \& S.V. Sánchez. 2000. Programa de Manejo Reserva de la Biosfera El Vizcaíno, México. Instituto Nacional de Ecología. México, D.F., México.

Carmona, R. 1995. Distribución temporal de las aves acuáticas en la playa "El Conchalito", Ensenada de La Paz, B. C. S. Inv. Mar. CICIMAR 10: 1-25.

Carmona, R. 2007. Dinámica poblacional y uso de hábitat de las aves playeras en la Ensenada de La Paz, como componente del Corredor Migratorio del Pacífico. Tesis de Doctorado, Universidad Autónoma de Baja California, Ensenada, BC., México.

Carmona, R. \& C. Carmona. 2000. Abundancia y riqueza especifica de aves playeras en la playa "El Conchalito", Baja California Sur, durante 1993 y 1995. Hidrobiológica 10: 69-78.

Carmona, R., G. Ruiz-Campos \& G. Brabata. 2004. Seasonal abundance of migrant shorebirds in Baja California Peninsula, Mexico, and California, USA. Wader Study Group Bull. 105: 65-70.
Carmona, R., G. Ruiz-Campos \& R. Vázquez. 2008a. Annotated checklist of shorebirds recorded at Ensenada de La Paz, Baja California Sur, Mexico. Wader Study Group Bull. 115: 140-147.

Carmona, R., N. Arce, V. Ayala-Pérez \& G.D. Danemann. 2008b. Abundance and phenology of Red Knots in the Guerrero Negro-Ojo de Liebre coastal lagoon complex, Baja California Sur, Mexico. Wader Study Group Bull. 115: 10-15.

Carmona, R., N. Arce, V. Ayala-Pérez \& G.D. Danemann. 2011. Seasonal abundance of shorebirds at the Guerrero Negro wetland complex, Baja California, Mexico. Wader study Group Bull. 118: 40-48.

Danemann, G.D. \& J.R. Guzmán-Poo. 1992. Notes on the birds of San Ignacio Lagoon, Baja California Sur, Mexico. West. Birds 23: 11-19.

Danemann, G.D., R. Carmona \& G. Fernández. 2002. Migratory shorebirds in the Guerrero Negro saltworks, Baja California Sur, México. Water Study Group Bull. 97: 36-41.

Donaldson, G., C. Ityslop, G. Morrison, L. Dickson \& I. Davidson. 2000. Canadian Shorebird Conservation Plan. Canadian Wildlife Service Special Publication, Ottawa, Canada.

Erwin, R.M., M. Coulter \& C. Howard. 1986. The use of natural vs man-modified wetlands by shorebirds and waterbirds. Col. Waterbird 9: 137-138.

Escalante, P., A.M. Sada \& J. Robles-Gil. 1996. Listado de nombres comunes de las aves de México. CONABIO-Sierra Madre, México.

Evans, P.R. 1976. Energy balance and optimal foraging strategies in shorebirds: some implications for their distributions and movements in the nonbreeding season. Ardea 64: 117-139.

Fernández, G., R. Carmona \& H. De la Cueva. 1998. Abundance and seasonal variation of Western Sandpipers (Calidris mauri) in Baja California Sur, Mexico. Southwest. Nat. 43: 57-61.

Fernández, G., H. de la Cueva, N. Warnock \& D.B. Lank. 2003. Apparent survival rates of Western Sandpiper (Calidris mauri) wintering in Northwest Baja California, Mexico. Auk 120: 55-61.

Frederick, P.C., B. Hylton, J.A. Heath \& M. Ruane. 2003. Accuracy and variation in estimate of large numbers of birds by individual observers using an aerial survey simulator. J. Field Ornithol. 74: 281-287.

Galindo, D. 2003. Uso de dos humedales, dulceacuícola y costero, por Calidris mauri (Caradriformes: Scolopacidae) al sur de la Península de Baja California, México. Tesis de Maestría, CICIMAR-IPN La Paz, Baja California Sur, México.

Grant, J. 1984. Sediment microtopography and shorebird foraging. Mar. Ecol. Prog. Ser. 19: 293-296. 
Gratto-Trevor, C.L. 2000. Marbled Godwit (Limosa fedoa), s.p. In A. Poole \& F. Gill (eds.). The Birds of North America, No. 492. The Birds of North America, Philadelphia, EE.UU.

Howell, S.N.G. \& S. Webb. 1995. A guide to Birds of Mexico and Northern Central America. Oxford University, Oxford, England.

Howes, J. \& D. Bakewell. 1989. Shorebirds Studies Manual. Asian Wetland Bureau. Publication No. 55. Kuala Lumpur, Malasia.

Huey, L.M. 1927. The bird life of San Ignacio and Pond Lagoons on the western coast of Lower California. Condor 29: 239-243.

Hsu, C.B., C.P. Chen \& H.L. Hsieh. 2009. Effects of sediment compaction on macroinfauna in a protected coastal wetland in Taiwan. Mar. Ecol. Prog. Ser. 375: 73-83.

Kelly, J.P. 2001. Distribution and abundance of winter shorebirds of Tomales Bay, California: implications for conservation. West. Birds 32: 145-166.

Lowther, P.E., H.D. Douglas III \& C.L. Gratto-Trevor. 2001. Willet (Catoptrophorus semipalmatus), s.p. In A. Poole \& F. Gill (eds.). The Birds of North America, No. 579. The Birds of North America, Philadelphia, EE.UU.

Massey, B.W. \& E. Palacios. 1994. Avifauna of the wetlands of Baja California, Mexico: current status, p 45-47. In J.R. Jehl Jr. \& N.K. Johnson (eds.). Studies in Avian Biology 15: A Century of Avifaunal Change in Western North American. Cooper Ornithol. Society. Lawrence, Kansas, EE.UU.

Melcher, C.P., A. Farmer \& G. Fernández. 2006. Version 1.1. Conservation Plan for the Marbled Godwit. Manomet Center for Conservation Science, Manomet, Massachussets.

Mellink, E., E. Palacios \& S. González. 1997. Nonbreeding waterbirds of the delta of the Rio Colorado, Mexico. J. Field Ornithol. 68: 113-123.

Molina, D., J. Torres-Guerrero \& M.L. Avelarde-Gómez. 2012. Riqueza de aves en el Área Natural Protegida Estero El Salado, Puerto Vallarta, Jalisco, México. Huitzil 13: 22-38.

Morrison, R.I.G., R.K. Ross \& M.S. Torres. 1992. Aerial surveys of Neartic Shorebirds wintering in Mexico: some preliminary results. Canadian Wildlife Sevice Program. Notes, 201, Ottawa, Canada.

Morrison, R.I.G., B.J. Mccaffery, R.E. Gill, S.K. Skagen, S.L. Jones, G.W. Page, C.L. Gratto-Trevor \& B.A. Andres. 2006. Population estimates of North American shorebirds, 2006. Wader Study Group Bull. 111: $67-85$.
Mouritsen, K.N. \& K.T. Jensen. 1992. Choice of microhabitat in tactile foraging dunlins Calidris alpine: the importance of sediment penetrability. Mar. Ecol. Prog. Ser. 85: 1-8.

Myers, J.P., R.I.G. Morrison, P.Z. Antas, B.A. Harrington, T.E. Lovejoy, M. Sallaberry, S.E. Senner \& A. Tarak. 1987. Conservation strategy for migratory species. Am. Sci. 75: 19-26.

National Geographic Society. 2002. Field guide to the birds of North America. National Geographic Society, USA.

O’Brien, M., R. Crossley \& K. Karlson. 2006. The shorebirs guide. Houghton Mifflin, Boston, EE.UU.

O’Reilly, K.M. \& J.C. Wingfield. 1995. Spring and autumn migration in Arctic shorebirds: some distance, different strategies. Am. Zool. 35: 222-233.

Page, G.W. \& E. Palacios. 1993. Pacific Flyway Project. Winter shorebird numbers in wetlands along the west coast of Baja California. PRBO and CICESE. Ensenada, B. C., México.

Page, G.W., E. Palacios, L. Alfaro, S. González, L.E. Stenzel \& M. Jungers. 1997. Numbers of wintering shorebirds in coastal wetlands of Baja California, México. J. Field Ornithol. 68: 562-574.

SEMARNAT. 2008. Estrategia para la conservación y manejo de las aves playeras y su hábitat en México. SEMARNAT, México, D.F., México.

SEMARNAT. 2010. Norma Oficial Mexicana NOM059-SEMARNAT-2010, Protección ambiental-Especies nativas de México de flora y fauna silvestres-categorías de riesgo y especificaciones para su inclusión o cambio-lista de especies en riesgo (segunda sección). Diario Oficial de la Federación.

Shepherd, P.C.F., L.J. Evans Orgen \& D.B. Lank. 2003. Integrating marine and terrestrial habitats in shorebird conservation planning. Wader Study Group Bull. 100: 40-42.

Skagen, S.K., B. Andres, S. Brown, G. Donaldson, B. Harrington, V. Johnston, S.L. Jones \& R.I.G. Morrison. 2003. Monitoring the shorebirds of North America: towards a inified approach. Wader Study Group Bull. 100: 102-104.

Sibley, D.A. 2000. National Audubon Society the Sibley guide to birds. Knopf, EE.UU.

Stenzel, L.E., C.M. Hickey, J.E. Kjelmyr \& G.W. Page. 2002. Abundance and distribution of shorebirds in the San Francisco Bay area. West. Birds 33: 68-98.

Tsipoura, N. \& J. Burger. 1999. Shorebird diet during spring migration stopover on Delaware Bay. Condor 101: 635-644. 
Verschuren, D., J. Tibby, K. Sabbe \& N. Roberts. 2000. Effects of depth, salinity, and substrate on the invertebrate community of a fluctuating tropical lake. Ecology 81: 164-182.

Warnock, N. 1994. Biotic and abiotic factors affecting the distribution and abundance of a wintering population of Dunlin. Tesis de Doctorado, University of California and San Diego State University, San Diego, California.

Wehtje, W. 2005. Identifying hybrid oystercatchers in southern California. West. Birds 36: 336-337.

Yates, M.G., J.D. Goss-Custard, S. McGrorty, K.H. Lakhani, S.E.A. Le V. Dit Durell, R.T. Clarke, W.E. Rispin, I. Moy, T. Yates, R.A. Plant \& A.J. Frost. 1993.
Sediment characteristics, invertebrate densities and shorebird densities on the inner banks of the Wash. J. Appl. Ecol. 30: 599-614.

Zar, J.H. 1999. Biostatistical Analysis. Prentice-Hall, Nueva Jersey, EE.UU.

Zárate-Ovando, B., E. Palacios, H. Reyes-Bonilla, E. Amador \& G. Saad. 2006. Waterbirds of the Lagoon Complex Magdalena Bay-Almejas, Baja California Sur, Mexico. Waterbirds 29: 350-364.

Zárate-Ovando, B., E. Palacios \& H. Reyes. 2008. Estructura de la comunidad y asociación de las aves acuáticas con la heterogeneidad espacial del complejo lagunar Bahía Magadalena-Almeja, Baja California Sur, México. Rev. Biol. Trop. 56: 371-389. 
